\title{
Two Imported Cases of New Variant COVID-19 First Emerging in Nigeria - Guangdong Province, China, March 12, 2021
}

\author{
Yao $\mathrm{Hu}^{1}$; Xiang $\mathrm{Zhao}^{2}$; Zhencui $\mathrm{Li}^{1}$; Min Kang ${ }^{1}$; Xiaoling Deng ${ }^{1}$; Baisheng $\mathrm{Li}^{1, \text { t* }}$
}

On February 19, 2021, a 48-year-old male (Case A, XG2275) returning from Nigeria and a 38-year-old female (Case B, XG2276) returning from Ukraine via airplane were tested by the laboratory of Guangzhou Customs using nose swabs to test for coronavirus disease 2019 (COVID-19). Both of them tested positive and were transported by ambulance from the isolation point to Guangzhou Eighth People's Hospital. They are still undergoing centralized medical observation in the hospital after recovery.

On March 5, Guangdong CDC received the samples and began virus isolation and genome sequencing analysis. On March 10, the 2 samples were sequenced using Nanopore GridION. On March 12, the sequencing analysis concluded that the 2 virus genomes belonged to lineage B.1.525, a new COVID19 variant first emerging in Nigeria, which was first detected by genome sequencing in mid-December in Nigeria but was also quickly found in cases in the United Kingdom, France, and elsewhere. As of March 8,2021 , a total of 577 counted sequences in 30 countries were found in the world (1).

Compared with the Wuhan reference sequence (EPI_ISL_402119) (2-3), the strain from Case A (XG2275) displayed 24 nucleotide variation sites (C241T, C1498T, A1807G, G2659A, C3037T, C6285T, T8593C, C9565T, C14407T, C14408T, C18171T, A20724G, A21717G, C21762T, G23012A, A23403G, G23593C, T24224C, C24748T, T26767C, C28308G, A28699G, $\mathrm{C} 28887 \mathrm{~T}$, and $\mathrm{G} 29543 \mathrm{~T}$ ) including the single nucleotide polymorphisms (SNPs) that defined the Llineage European branch and belonged to the Pangolin lineage B.1.525 (Figure 1). Furthermore, 10 amino acid mutation sites (E:L21X, M:I82T, N:A12G, N:T205I, S:Q52R, S:A67V, S:E484K, S:D614G, S:Q677H, and S:F888L) and 3 amino acid deletions (H69del, V70del, and Y144del) were detected in the protein that corresponded to the features of the Nigerian variant (B.1.525) (4).
Compared with the Wuhan reference sequence (EPI_ISL_402119) (2-3), the Case B (XG2276) strain displayed 22 nucleotide variation sites (C241T, C1498T, A1807G, C3037T, C6285T, T8593C, C9565T, C14407T, C14408T, C18171T, A20724G, C21762T, G23012A, A23403G, G23593C, T24224C, C24748T, T26767C, C28308G, A28699G, C28887T, and G29543T) including the SNPs that defined the L-lineage European branch and belonged to the Pangolin lineage B.1.525 (Figure 1). Furthermore, 9 amino acid mutation sites (E:L21X, M:I82T, N:A12G, N:T205I, S:A67V, S:E484K, S:D614G, S:Q677H, and S:F888L) and 3 amino acid deletions (H69del, V70del, and Y144del) were detected in the protein that corresponded to the features of the Nigerian variant (B.1.525).

The variant first emerging in Nigeria (B.1.525) shares the same 3 amino acid deletions (H69del, V70del, and Y144del) with the 501Y.V1 variant (also known as the B.1.1.7). The mutation $\mathrm{E} 484 \mathrm{~K}$ is also present in the 501Y.V2 variant first emerging in South Africa (also known as the B.1.351) and Brazilian strains (501Y.V3). The amino acid substitution at position $677(\mathrm{Q} 677 \mathrm{H})$ is identical to that found in variants recently described in the United States. The amino acid substitutions $\mathrm{Q} 52 \mathrm{R}$ and $\mathrm{A} 67 \mathrm{~V}$ are unique to the B.1.525 (4). This is the third recent detection of a major international variant following the detection of the United Kingdom 501Y.V1 variant and the South African 501Y.V2 variant. The transmissibility and pathogenicity of these mutant variants urgently needs further study $(5-6)$.

doi: $10.46234 / \mathrm{ccdcw} 2021.074$

\# Corresponding author: Baisheng Li, libsn@126.com.

\footnotetext{
${ }^{1}$ Guangdong Provincial Center for Disease Control and Prevention, Guangzhou, Guangdong, China; ${ }^{2}$ National Institute for Viral Disease Control and Prevention, China CDC, Beijing, China.
}

Submitted: March 15, 2021; Accepted: March 17, 2021 

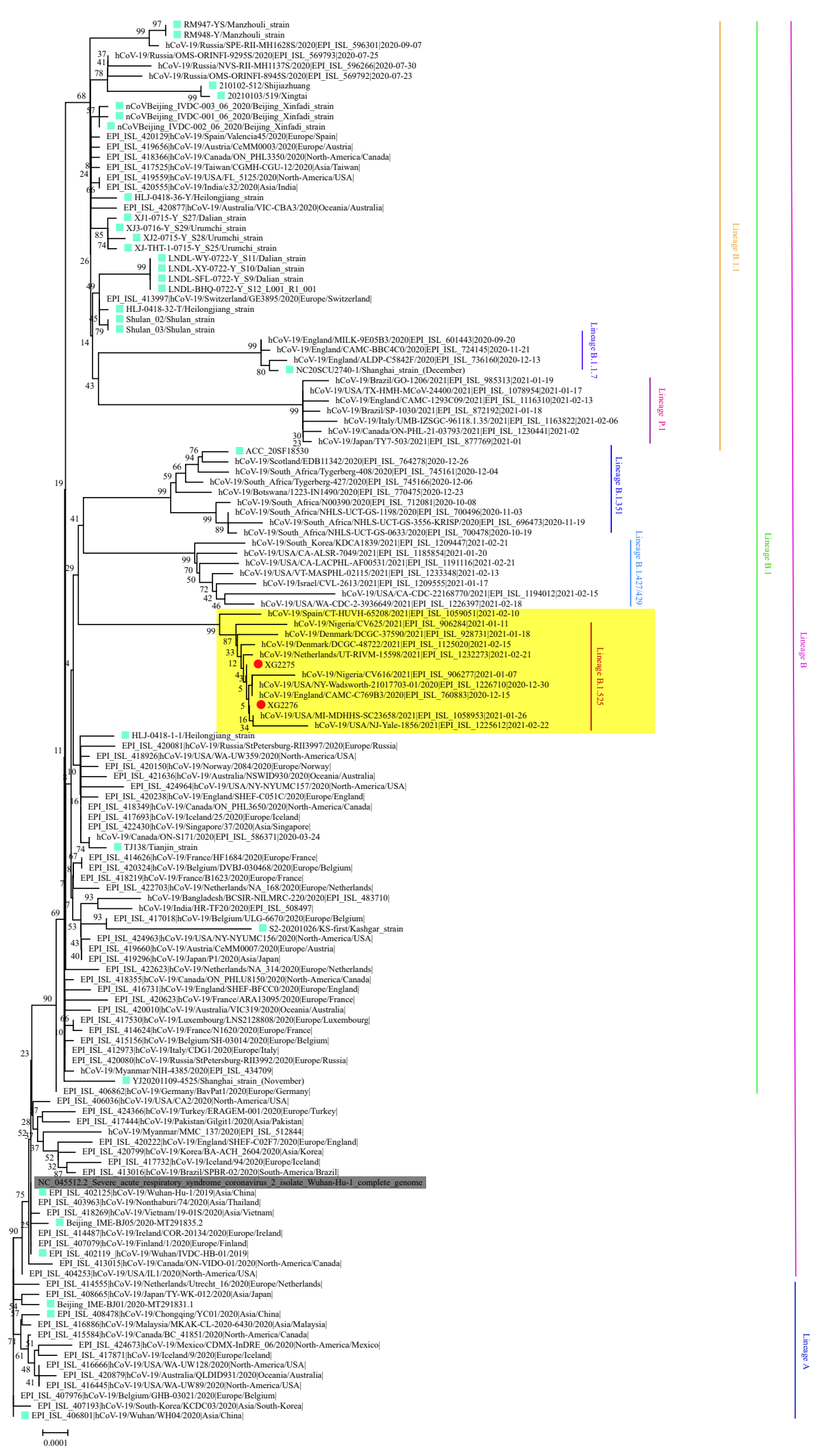

FIGURE 1. Phylogenetic tree based on the full-length genome sequences of the COVID-19 virus. The Nigerian variants (B.1.525) are highlighted in yellow and the Guangdong imported B.1.525 variant cases are marked with red dots. The strains associated with specific outbreaks in China are marked with blue squares. The Wuhan reference strain is shaded in gray. The 5 distinguished COVID-19 mutants are marked and colored on the right. The $L(A)$ - or $L(B)$-lineage and sublineages of the COVID-19 virus were marked and colored on the right. 


\section{REFERENCES}

1. GISAID. "GISAID hCOV-19 Tracking of Variants (see menu option ‘G/484K.V3 (B.1.525)')”. http://www.gisaid.org. [2021-03-08].

2. Tan WJ, Zhao X, Ma XJ, Wang WL, Niu PH, Xu WB, et al. A novel coronavirus genome identified in a cluster of pneumonia cases Wuhan, China 2019-2020. China CDC Wkly 2020;2(4):61-2. http://dx.doi.org/10.46234/ccdcw2020.017.

3. Wu F, Zhao S, Yu B, Chen YM, Wang W, Song ZG, et al. A new coronavirus associated with human respiratory disease in China. Nature 2020;579(7798):265-9. http://dx.doi.org/10.1038/s41586-020-2008-3.

4. William AH. A new COVID-19 variant from Nigeria raises increased concerns for containment and vaccination. forbes. https://www forbes.com/sites/williamhaseltine/2021/02/24/new-nigerian-variantcontinues-the-trend-of-dangerous-strains-threatening-covid-19-progress/ ?sh=1328d5864140. [2021-03-08].

5. Chen HY, Huang XY, Zhao X, Song Y, Hao P, Jiang H, et al. The first case of new variant COVID-19 originating in the United Kingdom detected in a returning student - Shanghai Municipality, China, December 14, 2020. China CDC Wkly 2021;3(1):1 - 3. http://dx. doi.org/10.46234/ccdcw2020.270.

6. Chen FJ, Li BS, Hao P, Song Y, Xu WB, Liu NK, et al. A case of new variant COVID-19 first emerging in South Africa detected in airplane pilot - Guangdong Province, China, January 6, 2021. China CDC Wkly 2021;3(2):28 - 9. http://dx.doi.org/10.46234/ccdcw2021.007. 